\title{
Controlling and creating plasmonic absorption processes in graphene nanostructures (presentation video)
}

Victor W. Brar, Min S. Jang, Michelle Sherrott, Seyoon Kim, Laura Kim, et al.

Victor W. Brar, Min S. Jang, Michelle Sherrott, Seyoon Kim, Laura Kim, Mansoo Choi, Harry A. Atwater, "Controlling and creating plasmonic absorption processes in graphene nanostructures (presentation video)," Proc. SPIE 9162, Active Photonic Materials VI, 91621C (17 August 2014); doi: 10.1117/12.2063844

EDIE Event: SPIE NanoScience + Engineering, 2014, San Diego, California, United States 


\title{
Controlling and creating plasmonic absorption processes in graphene nanostructures (Presentation Video)
}

\author{
Victor W. Brar ${ }^{1,2}$, Min S. Jang ${ }^{3}$, Michelle Sherrott ${ }^{1}$, Seyoon Kim ${ }^{1}$, Laura Kim ${ }^{1}$, Mansoo \\ Choi $^{3}$, Harry A. Atwater ${ }^{1}$ \\ 1. California Institute of Technology (United States) \\ 2. Kavli Nanoscience Institute (United States) \\ 3. Seoul National University (Republic of Korea)
}

\begin{abstract}
In this presentation, it will be shown that the plasmonic absorption of a graphene sheet can be enhanced and perturbed in controllable ways by controlling the thickness and permittivity of the supporting substrate. We will show the results of recent experiments where $25 \%$ absorption is achieved in the plasmonic modes of a graphene sheet by carefully selecting the properties of an underlying silicon nitride substrate. We also demonstrate how additional absorption pathways can be created by modifying the surrounding dielectric environment to have optical resonances that can couple to the graphene plasmons.

View presentation video on SPIE's Digital Library: http://dx.doi.org/10.1117/12.2063844.3879370894001
\end{abstract}

